\title{
Feasibility and clinical usefulness of the Unified Protocol in women survivors of violence
}

\author{
Jorge Osma $\mathbb{1}^{1} \cdot$ Alba B. Quilez-Orden ${ }^{1} \cdot$ Vanesa Ferreres-Galán ${ }^{2} \cdot$ María C. Meseguer $^{3} \cdot$ Silvia Ariza $^{3}$
}

Accepted: 31 December 2021

(c) The Author(s) 2022

\begin{abstract}
Violence against women is a serious public health problem. Worldwide, one in three women experiences violence throughout their lives. According to the triple vulnerability theory, being a survivor of violence could constitute a psychological vulnerability that would favor the appearance of emotional disorders, affecting their health, their style of parenting, and family well-being. The objective of this study is to verify the feasibility and usefulness of Unified Protocol (UP) in a group format for improving emotion regulation in women survivors of violence attended in the Specific Child and Adolescent Intervention Team (SCAIT), a social community service. The sample consists of 11 women who have experienced different types of violence who received the UP through 11 weekly, two-hour long and in group format sessions. Assessments were conducted at pre and post intervention and at 3 , 6, and 12 months follow-up. The results of the Multivariate Analysis of Variance (MANOVA) show an increase in quality of life (including family relationships), self-esteem and extraversion scores, and a reduction in interference (including family life), neuroticism, somatization, anxiety, emotional lack of control, negative affect, depression, and emotional rejection scores. This evolution of the scores with the MANOVA takes into account all the evaluation time points (post, and follow-up at 3, 6 and 12 months). The results were statistically significant $(p<0.05)$, and had large effect sizes $\left(\eta_{p}^{2}>0.14\right)$. In addition, the reliable change index was calculated to assess the change at an individual level of each of the participants for the different outcomes. $90.9 \%$ of the participants rated the quality of the program received as "excellent" and we obtained a high rate of session attendance $(82.64 \%)$. These results justify the need for randomized controlled clinical studies to demonstrate the feasibility and clinical efficacy of the UP in this context. This intervention would allow to address the needs of this population, by offering them comprehensive care and improving their mental health from a biopsychosocial model. Likewise, it might indirectly improve the well-being of the rest of the family members.
\end{abstract}

Keywords Emotional disorders $\cdot$ Unified Protocol $\cdot$ Social services $\cdot$ Violence against women $\cdot$ Family well-being

\section{Highlights}

- Being a survivor of violence increases the probability of developing an emotional disorder.

- Poor health status in mothers is associated with poorer parenting styles and family well-being.

- The Unified Protocol is an emotional-based CBT intervention that could improve mothers' mental health and family well-being.

- Unified Protocol shows preliminary viability and clinical utility for women who have experienced violence and have emotional disorders.

Emotional Disorders (hereinafter EDs) encompass anxiety, mood, and related disorders (Brown \& Barlow, 2009) and

Jorge Osma

osma@unizar.es

1 University of Zaragoza and Institute of Health Research of Aragón, Zaragoza, Spain are the most prevalent mental disorders worldwide (World Health Organization [WHO] 2017a). Focusing on the

2 Mental Health Unit of the Hospital Comarcal of Vinaròs, Castellón, Spain

3 Specific Child and Adolescent Intervention Team, Social Services of the City Council of Benicarló, Castellón, Spain 
world's female population, 178.7 million women suffer from at least one anxiety disorder (4.8\%), and 161.6 million from a mood disorder (4.4\%). In Spain, 1.8 million women suffer from at least one anxiety disorder $(7.8 \%)$, and 1.2 million from a mood disorder (5.4\%) (Institute for Health Metrics and Evaluation, 2017).

Brown and Barlow (2009) propose neuroticism (one of the "Big 5 personality traits") as a central dimension in the etiology, course, and maintenance of EDs and, in their triple vulnerability model, this variable conforms generalized biological vulnerability. In addition, the model includes two types of psychological vulnerability, one generalized (early experiences or parenting styles that contribute to a feeling of uncontrollability and unpredictability of events) and a specific one (adverse experiences undergone by the person, causing them to associate an intense emotional response with external or internal stimuli). For women, one of the main traumatic experiences they may undergo throughout their lives is the different types of violence against women, which can lead to generalized (e.g., family violence during childhood) or specific (e.g., intimate partner violence) psychological vulnerability. In addition, it has been shown how neuroticism scores are higher in women suffering violence than in control women (Brown et al., 2016), also increasing generalized biological vulnerability.

In Article 1 of the United Nations' "Declaration on the Elimination of Violence against Women", such violence is defined as "any act of violence based on female membership that results or may result in harm to women". Thus, different types of violence, such as intrafamilial, domestic, sexual, or gender violence, can be encompassed within this general definition (WHO, 2017b). In this article, we focus on women who have experienced different types of domestic violence. Domestic violence is defined as "physical, psychological, sexual or other abuse or aggression, inflicted by people in the family environment and generally directed at the most vulnerable members of the family: children, women and the elderly" (Ministerio de Sanidad y Consumo, 2003).

Due to the ambiguity of the definitions and the variability of concepts, it is difficult to clarify the extent of the issue (Larizgoitia, 2006), so that on many occasions the rates of violence vary depending on the definition adopted (Overstreet, 2000). Despite this, the figures show that violence against women is a serious public health problem that must be addressed. Worldwide, one in three women experiences violence throughout their lives. In Spain, 55 females suffering violence died in 2019 alone; 125,936 complaints were filed, and 68,714 phone calls were made to the telephone information and legal advice service on violence against women (Gobierno de España [Spanish Govt.] 2019).

The number of moderating variables that can contribute to the impact of violence is immense (Galovski et al., 2021).
There is a wide variety of sociocultural, historical, victim, and specific factors of trauma, which means that the impact of violence can vary substantially between individuals (Briere \& Jordan, 2004). One of the main consequences of experiencing violence is the development of mental health disorders, increasing their functional impairments and affecting their work, school, and social functioning (Helfrich et al., 2008). Some examples of functional impairment indicated by these authors include difficulties in finding or keeping a job, difficulties in continuing to attend work, problems concentrating, difficulties in maintaining friendships and interacting with other people, or difficulties in participating effectively in a group (Helfrich et al., 2008). In fact, women who experience violence are 2.3 times more likely to develop Post-Traumatic Stress Disorder (PTSD) than those who do not experience violence (Galovski et al., 2021). Also, the likelihood of experiencing symptoms like depression and anxiety (although anxiety and depressive responses can be sometimes an adaptative response to the environment) is higher in people exposed to violence compared to the normal population (e.g., Graham-Bermann et al., 2011) because, in the former, these disorders range between 30 and 60\% (Larizgoitia, 2006). But violence not only directly impacts the person who experiences it, but indirectly affects all family members and can result in a wide variety of negative consequences for children and family well-being (Herschell et al., 2017). As we have mentioned before, exposure to violence has an impact on the mental health of women who suffer it, and poor mental health in mothers is associated with an increase in the probability of developing behavioral difficulties in their children (D'Souza et al., 2019). In addition, being a survivor of violence tends to change parenting styles, increasing the probability of women survivors of violence adopting an overprotective style compared to other mothers (Boeckel et al., 2014), and this style is associated with poor self-regulation processes in children and adolescents (Christopher et al., 2013; Mak et al., 2020). Moreover, being a victim of violence is associated with difficulties in communicating with children, since mothers avoid talking about certain topics with them with the intention of protecting them (Kamody et al., 2020). Because of all of these consequences, the treatment of EDs in mothers with risk factors (e.g., history of violence) can be a very positive way to improve parenting styles and family well-being (Carreras et al., 2019).

To better understand the etiology and maintenance of EDs, Bullis et al. (2019) describe the Functional Model of EDs. This model is composed of three related components: (1) the tendency to experience negative emotions (e.g., anxiety and depression); (2) aversive reactions to emotional experiences when they occur, and (3) efforts to suppress emotional experiences or, if these don't work, avoid them. What this model explains is that EDs are characterized by aversive reactions to emotional experiences, which generally implies avoidant coping 
(Bullis et al., 2019). It is suggested that all these components are triggered by a personality dimension called neuroticism (Barlow et al., 2014).

As in the general population (Brown et al., 2001), in female survivors of violence, EDs also present with high levels of comorbidity, that is, more than one psychological disorder at a time, reaching $84.2 \%$ for PTSD and depression (Paz et al., 2004). This comorbidity makes it difficult to choose the most appropriate psychological intervention. Although there is evidence of the effectiveness of Cognitive Behavioral Therapy (hereinafter CBT) for the specific treatment of EDs (Trabold et al., 2018), there is still a limitation regarding the most appropriate treatment when there is comorbidity. In addition, some females that have experienced violence have unspecified anxiety or mood disorders, or subclinical symptoms, further complicating treatment choice. In this sense, the Unified Protocol for the Transdiagnostic Treatment of EDs (hereinafter, UP) is a CBT-based intervention designed to treat the etiological and maintenance mechanisms of EDs (Boettcher \& Conklin, 2017; Kennedy \& Barlow, 2017). Differents studies suggest the importance of etiological and transdiagnostic framing for intervention with violence-exposed populations (e.g., Miller-Graff and Howell, 2016). The UP consists of 8 treatment modules, 5 of which are considered core modules because are used to train a specific emotional regulation skill (Sauer-Zavala et al., 2017). Despite the UP being a protocolized intervention, it is also flexible, which allows clinicians to be able to use only some of the modules or to change their order to personalize the UP to their patients (Sauer-Zavala et al., 2019). The UP aims to provide adaptive emotion regulation strategies so that individuals can accept, tolerate and respond effectively to intense emotions without giving up their personal goals and objectives (Barlow et al., 2018).

This intervention focuses on a wide range of emotional psychopathology and subclinical or unspecified symptoms, thus reducing treatment times and costs, and enhancing response to treatment (Barlow et al., 2017). In addition, the UP can be applied in group format (e.g., Osma et al., 2021), promoting social support among the participants, an aspect that has proven to be a protective factor for a good prognosis in female survivors of violence (Buesa \& Calvete, 2013), and that has obtained better results of efficiency than the individual format (Echeburúa et al., 2014). To date, the UP has shown significant improvement in pre-treatment symptoms and has obtained effect sizes that are at least comparable to existing specific CBT protocols, in face-to-face, group, and online format (e.g., Sakiris \& Berle, 2019).

It is important to highlight that the majority of the positive outcomes on the feasibility and efficacy of the UP for the treatment of EDs have been achieved in clinical and healthcare settings, not in community settings. This fact is an important gap because when women go to healthcare settings they do not usually declare themselves as having experienced violence (Bradley et al., 2002). Moreover, less stigma regarding psychological disorders has been detected in community settings (Muñoz, 2009). In addition, we have found no studies on the clinical usefulness of the UP for improving anxiety and depressive symptoms in women who have experienced or currently experience violence.

Considering the three-axis analysis described by Ferro and Vives (2004), we need evidence-based interventions not only regarding their clinical effectiveness, changes in the expected direction, and maintenance over time (axis of efficacy), but also interventions that are cost-effective for the resource that applies to them (i.e., group interventions; axis of efficiency). Finally, we also need interventions accepted and positively valued by the users who receive them (axis of effectiveness). Therefore, the general objective of this study is to explore the feasibility and clinical usefulness of a group adaptation of the UP, applied in a community context to a group of women who had experienced violence. The specific objectives pursued are: [1] to obtain a statistically significant reduction in the scores of depression, anxiety, neuroticism, negative affect, somatization, panic, emotional lack of control, emotional rejection, lack of emotional attention, emotional confusion, and interference of symptoms in the participants' lives after the intervention; [2] to obtain a statistically significant increase in scores in participants' extraversion, positive affect, self-esteem, and quality of life after the intervention; [3] and to confirm that the changes in both directions are maintained over time, at three temporal moments that involve follow-up at three, six, and twelve months. In addition, we intend [4] to obtain data that show the participants' acceptance and satisfaction with the intervention (high rates of session attendance and high scores in satisfaction with treatment).

Based on these objectives, we established the following study as a single-arm pilot study to explore the feasibility and clinical usefulness of a group application of the UP in a social services context.

\section{Method}

\section{Participants}

The total sample of this study consisted of 11 participants, all women, with a mean age of 49.64 years $(S D=5.52$, range $=$ 40-59), users of the "Equipo Específico de Intervención con 
Infancia y Adolescencia" [Specific Child and Adolescent Intervention Team (hereinafter, SCAIT)] at Social Services Center of Benicarló (Castellón, Spain). Ten of the participants have Spanish nationality and 1 has Moroccan nationality; 6 participants are divorced and 5 are in a couple; and all of them have children, between 1 and 3. At the clinical level, 5 participants presented subclinical anxious-depressive symptoms without medication, 1 of them had an unspecified depressive condition with medication, and 5 had an unspecified anxietydepression condition with medication. One of the participants (Participant 2) suffers from fibromyalgia. She is the only participant with a significant medical condition. The main conflicts that the women refer to are problems in managing the behavior of the children in the case of 7 participants, filio-parental violence in the case of 3 of them, and a history of intimate partner violence in the case of 1 participant. Regardless of the conflict addressed by the SCAIT, all the participants have experienced domestic violence, by their husbands (6 participants) or their children (4 participants). It should be noted that most of the participants highlight the management of their children's behavior as the main problem, something to be expected considering that they are users of a social service program whose aim is to address the needs of children and families, serving families with high-risk factors to improve their wellbeing.

\section{Procedure}

The sample of this study was a convenience sample obtained from the SCAIT service at Social Services Center of Benicarló (Castellón, Spain). The SCAIT is a municipal service, included within the social care network as a resource directed at the sectors of family and children. It has interdisciplinary, specific, and specialized teams, whose goal is the prevention, care, and treatment of situations of crisis and family destructuring (Sospedra et al., 2010). This service offers psychosocial counseling, therapy, and family mediation services (Consellería de Bienestar Social [Ministry of Social Welfare] 2014).

The SCAIT psychologist (M.C.M.) was responsible for informing the users of the existence of this study, and of the characteristics of the intervention to be performed. She was also responsible for carrying out the initial evaluation of the users at the Social Services Center of Benicarló (Castellón, Spain). Participation in the study was voluntary (participants' intention to treat) and without financial compensation. The inclusion criteria were being of legal age; speaking Catalan or Spanish fluently; committing to attend the sessions; presenting anxious or depressive symptomatology or meeting the criteria for at least one $\mathrm{ED}$, and understanding and accepting the contents of the informed consent, expressed by signing it. Exclusion criteria were presenting a serious mental disorder (bipolar disorder, schizophrenia, or organic mental disorder); a personality disorder; being in the process of grieving; substance abuse, or presenting a suicide risk at the time of evaluation. After selection according to the inclusion criteria, the participants were assigned to two different intervention shifts (morning and afternoon) depending on their availability. In total, five evaluations were performed at different times: pre-intervention, post-intervention, and follow-ups at three, six, and twelve months.

To ensure the proper implementation of the UP, the psychologists of the morning and afternoon intervention groups (V.F.G. and M.C.M.) received a 20-hour training course given by a psychologist accredited by the Unified Protocol Institute as UP trainer (J.O.) and were continuously supervised by him during treatment. The study was approved by the Ethics and Research Committee of the Hospital Comarcal de Vinaròs (Castellón, Spain).

\section{Intervention}

The intervention consisted of 11 weekly, two-hour sessions and in group format to address seven UP modules. For module 1, "Setting goals and maintaining motivation", a single session was used; two sessions were used for module 2, "Understanding emotions"; a single session was used for module 3, "Mindful emotional awareness"; three sessions were used for module 4, "Cognitive flexibility", which was the most difficult to understand for the participants; a single session was used for module 5, "Opposing emotional behaviors". Module 6, "Understanding and coping with physical sensations", was eliminated because the participants manifested on several occasions that physical sensations were not associated with anxiety/fear or other overwhelming emotions. Although the content about the relationship between physical sensations, thoughts, and behaviors was explained, the interoceptive exposure exercises were not practiced in this case. It is important to note that, although the interoceptive exposure was not practiced in this group, it is necessary to assess whether these associations exist, because they are quite frequent. If they exist, this module should be developed, as it can be very beneficial for the participants.

Two sessions were used for module 7, "Emotional exposure"; and, finally, a single session was used for module 8, "Recognize achievements and look to the future". In this study, we provided a special emphasis on the validation of the participants' emotional experience, as they had been exposed to constant situations of invisibilization and invalidation. Some examples of these situations of 
invalidation described by the participants are: lack of respect on the part of their children or partners; feelings of not being good mothers or not having known how to educate their children well, blaming themselves for the behavior of their children; hiding situations of violence experienced in the family context to protect their family or due to fear of the consequences; etc. In addition, their emotions are congruent with the situation of violence they are experiencing (anxiety and sadness), but their closest environment invalidates them (e.g., you should not feel anxious or sad or you should be happy with your family).

\section{Instruments}

Mini-International Neuropsychiatric Interview (MINI; Sheehan et al., 1998; Spanish version by Ferrando et al., 2000)

It contains structured questions that evaluate major psychiatric disorders and it is divided into 16 modules, each with structured questions corresponding to the different diagnostic categories. In the validation study, it had a value of 0.75 for Cohen's Kappa, showing good interjudge reliability.

Rosenberg Self-Esteem Scale; (RSES; Rosenberg, 1965; Spanish version by Vázquez et al., 2004)

It contains 10 items in the form of sentences with a 4-point Likert scale ranging from 1 (strongly agree) to 4 (strongly disagree). Higher scores indicate a higher level of selfesteem. In the present study, we obtained a Cronbach alpha of 0.69 .

\section{Quality of Life Index (QLI; Mezzich et al., 2000)}

It contains 10 items that are rated on a 10-point Likert scale ranging from 1 (Poor) to 10 (Excellent). Those items are: physical well-being, emotional well-being, self-care, and independent functioning, occupational functioning, interpersonal functioning (it includes family functioning), social-emotional support, community and services support, personal fulfillment, spiritual fulfillment, and overall quality of life. The higher the score, the higher the quality of life. In the present study, we obtained a Cronbach alpha of 0.88 .

\section{Maladjusment Scale (MS; Echeburúa et al., 2000)}

This scale reflects the extent to which the person's current problems are affecting the different areas of their daily life, which are scored on a 6-point Likert scale ranging from 0 (not at all) to 5 (Very severe). These areas are: work, social life, free time, relationship with partner, family life, and overall maladjustment in everyday life. The higher the score, the greater the interference of current problems in the person's life. We found a Cronbach alpha of 0.80 in this study.

Positive and Negative Affect Scale (PANAS; Watson et al., 1988; Spanish version by Sandín et al., 1999)

It consists of 20 items that measure both positive and negative affect, 10 items for each dimension. Each item is rated on a 5-point Likert scale ranging from 1 (Not at all, very slightly) to 5 (Extremely). The higher the score in each of the dimensions, the greater the affect evaluated. In the present study, we found a Cronbach alpha of 0.90 for Positive Affect and of 0.96 for Negative Affect.

Brief Symptom Inventory (BSI-18; Derogatis, 2001; Spanish version by Andreu et al., 2008)

It is made up of 18 items and screens psychiatric symptoms by means of a 5-point Likert scale ranging from 0 (Not at all) to 4 (Very much). The higher the score in each of the subscales (Somatization, Depression, Anxiety, and Panic), the greater the severity of the symptomatology. Cronbach alpha scores in the present study were 0.63 for the Somatization, 0.88 for Depression, 0.78 for Anxiety, and 0.69 for Panic subscales.

NEO-FFI Personality Inventory (NEO-FFI; Costa and McCrae, 1999)

This contains 60 items that provide a quick and overall measurement of the five major personality factors. The responses are rated on a 5-point Likert scale ranging from 0 (Strongly agree) to 4 (Strongly disagree). The higher the score in each of the dimensions, the higher the tendency toward that personality factor. In this study, we only used Neuroticism and Extraversion factors, with Cronbach alpha values of 0.66 and 0.86 , respectively.

\section{Difficulties in Emotion Regulation Scale (DERS; Gratz and} Roemer, 2004; Spanish version by Hervás and Jódar, 2008)

This scale includes 28 items rated on a 5-point Likert scale ranging from 1 (almost never $/ 0-10 \%$ of the time) to 5 (almost always/90-100\% of the time). The higher the total score, the higher the emotional dysregulation. In this study, the DERS obtained Cronbach alpha values of 0.92 for Emotional Lack of Control, 0.94 for Emotional Rejection, 0.79 for Life Interference, 0.92 for Lack of Emotional Attention, and 0.61 for Emotional Confusion. 
Questionnaire for the evaluation of the UP components (ad hoc, see "Appendix 1")

It consists of 9 items that evaluate the extent to which the participants considered that the UP in general and each of its components, in particular, were useful to help them regulate their emotions adaptively, rated on a 10-point Likert scale ranging from 0 (Not at all) to 10 (very much). The higher the score, the better the UP rating.

\section{Satisfaction with treatment questionnaire (ad hoc)}

This scale presents 7 items that evaluate participants' overall satisfaction with the treatment, based on its perceived quality, adaptation to their expectations, their recommendation to friends or family, the usefulness of the techniques learned, their joint and general satisfaction with the treatment, the discomfort it generated, and the likelihood that they would choose such a treatment again. Participants rated the items on a 4-point Likert scale ranging from 0 (Bad/Not at all) to 4 (Excellent) Very much). The higher the score, the greater the satisfaction with the treatment.

\section{Survey about the opinion of the treatment received (ad hoc)}

Through a survey developed specifically for this study, participants were asked three open questions that they were requested to answer in writing. The first question refers to the objectives they set out to achieve, the second asked them which objectives they had achieved or was in the process of achieving, and the third refers to their beliefs about the benefits provided by the intervention.

\section{Data analysis}

The analyses were carried out with the statistical package IBM SPSS Statistics version 22.0 for Windows (IBM Corp., 2013). First, the sociodemographic characteristics of the total sample $(N=11)$ were analyzed with descriptive statistics, calculating the mean and standard deviation of the scores in the different questionnaires. Next, a Missing Values Analysis and Little's MCAR test were performed to determine the random distribution of missing values, and after confirming this, the Last Observation Carried Forward (LOCF) was used. Internal consistency was then explored using Cronbach's alpha.

As the sample size is less than $50(N=11)$, the normal distribution of the variables was verified with the Shapiro-Wilk normality test. A Multivariate Analysis of
Variance (MANOVA) was then performed to check whether the differences in the scores of the variables measured at different times were statistically significant. Finally, the Reliable Change Index (hereinafter RCI), which evaluates the clinically significant change obtained to determine in which variables the scores approach those of the normative sample, was calculated. The RCI is a procedure that assesses whether the change is reliable, beyond the fluctuation associated with imprecise measurement tools. A clinically significant change will be considered if an RCI score is equal to or greater than 1.96 (Jacobson \& Truax, 1991).

Session attendance was also recorded, to calculate the attendance rate and use it as an indicator of feasibility and acceptance of treatment. For the same purpose, quantitative and qualitative analyses were carried out. At the quantitative level, the participants' assessment of and satisfaction with the treatment received was evaluated through two questionnaires. Qualitatively, participants were asked to answer three main questions about the treatment, and their answers were analyzed by selecting and sorting information through a process of segmentation, identifying key topics, and categories of analysis (De Andrés Pizarro, 2000).

\section{Results}

\section{Descriptive analyses}

The results of descriptive analyses for the sociodemographic characteristics of the sample are described in the participants section. The mean scores and standard deviations of all the administered instruments are shown in Table 1.

The Missing Value Analysis showed that $6.24 \%$ of the data were missing from the total set of all the variables. Little's MCAR test showed a significance of $p=1.00(p>$ 0.05), which implies a random distribution of the missing values, so LOCF was performed for those missing values. The results of the Shapiro-Wilk normality test showed that the scores of the participants in the different variables were normally distributed $(p>0.05)$.

\section{Clinical Utility}

In this section, the results directly related to objectives 1 , 2 , and 3 will be addressed. MANOVA showed that the statistically significant changes were increased scores on the variables of quality of life, including the quality of the interpersonal area (family), self-esteem, extraversion, and decreased scores on the variables of interference 
Table 1 Mean and standard deviation (SD) of the sample scores on the instruments

\begin{tabular}{|c|c|c|c|c|c|c|c|c|c|c|c|c|}
\hline \multirow[t]{2}{*}{ Variable } & \multicolumn{2}{|c|}{ PRE } & \multicolumn{2}{|c|}{ POST } & \multicolumn{2}{|c|}{$\begin{array}{l}\text { 3-MONTH } \\
\text { FOLLOW-UP }\end{array}$} & \multicolumn{2}{|c|}{$\begin{array}{l}\text { 6-MONTH } \\
\text { FOLLOW-UP }\end{array}$} & \multicolumn{2}{|c|}{$\begin{array}{l}\text { 12-MONTH } \\
\text { FOLLOW-UP }\end{array}$} & \multicolumn{2}{|l|}{ MANOVA } \\
\hline & $N$ & $M(S D)$ & $N$ & $M(S D)$ & $N$ & $M(S D)$ & $N$ & $M(S D)$ & $N$ & $M(S D)$ & $F$ & $\eta_{p}^{2}$ \\
\hline RSES & 11 & $26.73(4.60)$ & 11 & $30.27(4.08)$ & 11 & $30.91(5.07)$ & 11 & $30.27(4.24)$ & 10 & $31.10(3.98)$ & $4.025 * *$ & 0.309 \\
\hline QLI & 11 & $5.55(1.53)$ & 11 & 7.19 (1.48) & 11 & $7.10(1.24)$ & 11 & $6.92(0.92)$ & 10 & $7.23(1.82)$ & $5.346 * *$ & 0.373 \\
\hline QLI-Interpersonal & 11 & $6.36(2.11)$ & 11 & $7.73(1.27)$ & 11 & $7.18(1.89)$ & 11 & $7.36(1.57)$ & 10 & $7.70(1.83)$ & $2.848 *$ & 0.240 \\
\hline MS & 11 & $16.09(6.11)$ & 11 & $8.45(6.53)$ & 11 & $7.73(5.80)$ & 11 & $7.55(6.15)$ & 10 & $9.40(6.80)$ & $10.999 * * *$ & 0.550 \\
\hline MS-Family Life & 11 & $2.36(1.03)$ & 11 & $1.91(1.38)$ & 11 & $1.27(1.01)$ & 11 & $0.91(1.04)$ & 10 & $1.70(1.06)$ & $5.159 * *$ & 0.364 \\
\hline PANAS-Positive & 11 & $28.73(8.33)$ & 11 & $36.64(6.61)$ & 11 & $33.36(8.65)$ & 11 & $31.10(8.43)$ & 10 & $30.40(9.94)$ & 2.387 & 0.210 \\
\hline PANAS-Negative & 11 & $31.55(11.40)$ & 11 & $23.73(9.34)$ & 11 & $20.00(8.66)$ & 11 & $17.82(5.76)$ & 10 & $20.60(7.00)$ & $5.822 * *$ & 0.393 \\
\hline BSI-18-Somatization & 11 & $10.00(4.80)$ & 11 & $5.45(4.57)$ & 11 & $2.36(2.34)$ & 11 & $1.82(2.36)$ & 10 & $4.30(6.00)$ & $9.839 * * *$ & 0.522 \\
\hline BSI-18-Depression & 11 & $10.18(6.51)$ & 11 & $5.64(5.01)$ & 11 & $3.55(3.08)$ & 11 & $2.73(1.68)$ & 10 & $3.80(4.83)$ & $6.002 * *$ & 0.400 \\
\hline BSI-18-Anxiety & 11 & $6.18(3.19)$ & 11 & $4.36(3.04)$ & 11 & $2.45(2.07)$ & 11 & $2.00(1.95)$ & 10 & $2.20(2.39)$ & $7.397 * * *$ & 0.451 \\
\hline BSI-18-Panic & 11 & $5.73(3.32)$ & 11 & $3.09(3.48)$ & 11 & $0.45(0.69)$ & 11 & $0.82(0.87)$ & 10 & $1.30(2.31)$ & $13.678 * * *$ & 0.603 \\
\hline DERS-Total & 11 & $77.27(20.12)$ & 11 & 59.55 (19.79) & 11 & 51.27 (12.59) & 11 & $48.82(9.21)$ & 10 & $50.30(15.29)$ & $9.302 * * *$ & 0.508 \\
\hline $\begin{array}{l}\text { DERS-Lack of Emotional } \\
\text { Attention }\end{array}$ & 11 & $11.00(4.31)$ & 11 & $8.91(3.11)$ & 11 & $10.00(4.00)$ & 11 & $10.00(3.32)$ & 10 & $9.80(3.46)$ & 1.391 & 0.134 \\
\hline DERS-Confusion & 11 & $10.82(3.22)$ & 11 & $8.27(3.44)$ & 11 & $7.64(4.76)$ & 11 & $6.82(2.44)$ & 10 & $7.40(2.99)$ & 2.212 & 0.197 \\
\hline DERS-Rejection & 11 & $20.45(7.88)$ & 11 & $15.55(7.65)$ & 11 & $11.18(3.97)$ & 11 & $13.00(7.44)$ & 10 & $12.70(7.86)$ & $5.317 * *$ & 0.371 \\
\hline DERS-Interference & 11 & $11.27(3.55)$ & 11 & $9.00(4.49)$ & 11 & $7.91(2.81)$ & 11 & $6.73(1.55)$ & 10 & $7.10(3.18)$ & $5.215 * *$ & 0.367 \\
\hline DERS-Lack of Control & 11 & $23.73(9.34)$ & 11 & $17.82(7.15)$ & 11 & $14.55(3.98)$ & 11 & $12.27(3.64)$ & 10 & $13.30(6.22)$ & $7.139 * *$ & 0.442 \\
\hline NEOFFI-Neuroticism & 11 & $29.45(6.58)$ & 11 & $23.18(7.61)$ & 11 & $22.00(8.87)$ & 11 & $19.09(8.32)$ & 10 & $19.50(9.68)$ & $10.340 * * *$ & 0.535 \\
\hline NEOFFI-Extraversion & 11 & $26.55(8.29)$ & 11 & $30.91(7.93)$ & 11 & $30.45(9.21)$ & 11 & $31.18(8.77)$ & 10 & $29.40(9.17)$ & $2.849 *$ & 0.240 \\
\hline
\end{tabular}

Results of the multivariate analysis of variance (MANOVA)

PRE: pre-intervention; POST: post-intervention; RSES: Rosenberg Self-Esteem Scale; QLI: Quality of Life Index; MS: Maladjustment Scale; PANAS: Positive and Negative Affect Scale; BSI-18: Brief Inventory of Symptoms; DERS: Difficulties in Emotion Regulation Scale; NEOFFI: NEOFI Personality Inventory; after the name of the scale, the name of the subscales appears separated by a hyphen; * $p<0.05 ; * * p<0.01 ; * * * p<$ $0.001 ; \eta_{\mathrm{p}}^{2} \simeq 0.01 \rightarrow$ small effect size; $\eta_{\mathrm{p}}^{2} \simeq 0.06 \rightarrow$ medium effect size; $\eta_{\mathrm{p}}^{2}>0.14 \rightarrow$ large effect size

(including interference in family life), neuroticism, somatization, anxiety, emotional dysregulation, emotional lack of control, negative affect, depression, and emotional rejection $(p<0.05)$. The increase in positive affect and the decrease in the variables lack of emotional attention and emotional confusion did not show statistically significant changes $(p>0.05)$. In all the variables, the effect sizes were large $\left(\eta_{p}^{2}>0.14\right)$. Table 1 depicts the mean scores on each variable and assessment time, and the specific value of the statistic, effect size, and significance.

The results of the RCI showed that, after the end of the intervention, ten participants $(90.91 \%)$ maintained normalized scores in the variable interference of symptomatology; eight participants $(72.73 \%)$ maintained normalized scores in the variable of emotional dysregulation; seven participants $(63.64 \%)$ maintained normalized scores in the variables quality of life, positive affect, and negative affect; six participants $(54.55 \%)$ did so in the variables quality of the interpersonal area and rejection of emotions; five participants $(45.45 \%)$ did so in the variable emotional lack of control; four participants $(36.36 \%)$ did so in the variables self-esteem, interference of emotions, neuroticism and depression; three participants $(27.27 \%)$ did so in emotional confusion; two participants (18.18\%) maintained normalized scores in interference in family life, emotional lack of attention, somatization and extraversion, and one participant $(9.09 \%)$ maintained normalized scores in interference in somatization and anxiety.

The results of the RCI showed that one year after the end of the intervention, eight participants $(72.73 \%)$ maintained normalized scores in the variable emotional dysregulation; seven participants (63.64\%) maintained normalized scores in the variables quality of life, emotional lack of control, somatization and anxiety; six participants $(54.55 \%)$ did so in the variables quality of the interpersonal area, interference of symptomatology, positive affect, negative affect, rejection and interference of emotions, neuroticism, somatization and depression; five participants $(45.45 \%)$ did so in the variable selfesteem; four participants $(36.36 \%)$ did so in the variable 


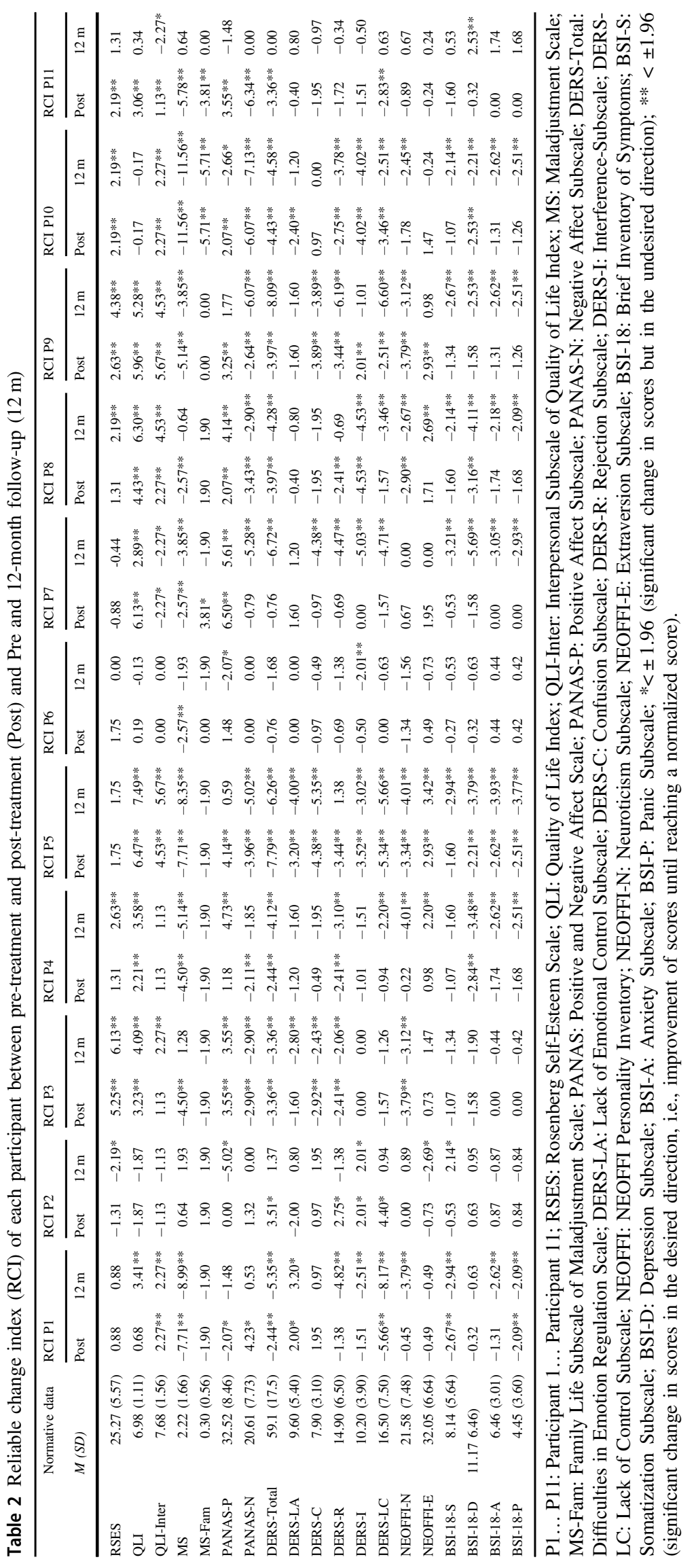


Table 3 Coding of the participants' responses to the satisfaction survey on the treatment received

What objectives did you set out to achieve during this intervention?

1. Emotional regulation (7 participants): "My main objective was to understand my fear", "I want to learn to relax"

2. Increased self-esteem and quality of life (6 participants): "I need to strength my self-esteem", "I need not to feel self-conscious"

3. Improve family well-being ( 3 participants): "I want to achieve a more positive relationship with my family and with myself"

4. Improve parenting styles ( 2 participants): "I want to learn to better educate my children, ask them respectfully and that they respect me" 5. Reduction on the interference of intense emotions (5 participants): "Being able to separate all the problems of my daily life, because I only have time to be aware of them"

What objectives have you achieved, or are you in the process of achieving?

1. Greater knowledge about the emotional response (4 participants): "I have learned to identify thoughts, to observe the physical sensations in my body, what behaviors I usually follow to face the intense emotions and also I have learned to identify what factors trigger this fear that I feel and the consequences of my behaviors", "I have learned to differentiate my emotions"

2. Reduction on the frequency, duration and emotional intensity (9 participants): "It has helped me to feel less anxiety", "Before, the anxiety lasted for at least an hour, now it doesn't"

3. Learning about anxiety management and mindfulness techniques (8 participants): "Now I am able to calm myself down", "I always keep in mind not judging", "Focus on that present moment"

4. Emotional exposure (5 participants): "Now I am able to read the emails that my lawyer asks me to"

5. Generate alternative thoughts and carry out opposite behaviors to those driven by emotions (7 participants): "When I raise the tone of voice I realize it, and I can change it to a softer tone", "I have managed to think before acting", "I have learned to not think that everything will always go wrong", "I have more control of my impulses and this make me not see things so negatively" 6. Increase self-esteem and empowerment (7 participants): "Now I know that if I want to do something, I am able to do it", "Until now I was always walking and looking behind me, now I don' $t$ "

Why would you say that attending these sessions has helped you?

1. Increased self-esteem, self-confidence, and motivation (3 participants): "Now I feel that I am a useful and optimistic person", "I should start loving myself", "You have to keep working", "To know that I can achieve my aims has given me the confidence to face the problems in a better way, even if it requires effort"

2. Improvement in general level of health (4 participants): "Now I sleep well", "Before I felt pain in my body or my hands", "Before, I always went to the doctor because of my asthma, now I don't need it" 3. Increased perception of social support (9 participants): "I have always felt very good here", "When everyone believes you, it is a liberation", "Here I have been able to tell what I have kept quiet during years" 4. Increase in quality of life (7 participants): "All this has given me my life back", "I am very grateful, it has given me my life again"

5. Increase family well-being (6 participants): "Now I am happy, and my family too"

6. Favor a sensitive parenting style (2 participants): "Now I speak to my son in a calmer way, and when I raise the tone of voice, I realize it and I can change it to a softer tone", "My son listens to me, understands me and does what I ask", "My son and I understand each other better and respect each other more", "This therapy has helped me a lot with my parenting skills"

7. Reduce problem behaviors in children (2 participants): "Now my daughter has fewer tantrums and they are less intense", "My daughter takes orders more, that's a great achievement" emotional confusion; three participants $(27.27 \%)$ did so in extraversion; two participants (18.18\%) maintained normalized scores in lack of emotional attention, and one participant $(9.09 \%)$ maintained normalized scores in interference in family life. Table 2 shows the RCI values between pretest and posttest, and between pretest and the 1 -year follow-up, respectively.

\section{Viability and satisfaction with the intervention}

In this section, the results directly related to objective 4 will be addressed. Participants attended a mean number of sessions of $9.09(S D=1.38$, range $8-11)$ of the 11 sessions that made up the treatment, which is $82.64 \%$ of the program. In addition, no dropouts were recorded during treatment or at the follow-ups.

In the UP component assessment questionnaire, the mean score obtained was 9 for the UP in general $(S D=1.34$, range $=6-10$ ). Focusing on each of the techniques, the scores varied between 8.18 and 8.91. The "understanding emotion" technique obtained a mean score of $8.73(S D=$ 1.49 , range $=5-10$ ); the "mindful emotional awareness" technique a mean score of $8.64(S D=1.63$, range $=6-10)$; the "cognitive flexibility" technique a mean score of 8.32 $(S D=1.85$, range $=5-10)$; the "opposing emotional behaviors" technique a mean score of $8.55(S D=1.52$, range $=$ $6-10)$; and the "emotional exposure" technique a mean score of $8.55(S D=1.51$, range $=6-10)$.

In the treatment satisfaction questionnaire $(N=11)$, $90.9 \%$ of the participants $(n=10)$ rated the quality of the program received as "excellent" and the remaining participant rated it as "good". A $54.5 \%(n=6)$ stated that the intervention "totally" corresponded to what they expected, while the remaining 5 participants stated that it did but "partially". To the question "If a friend or family member needed similar help, would you recommend this treatment program?", 90.9\% of the participants $(n=10)$ answered "Yes" and the remaining participant answered "probably yes". Lastly, $81.8 \%$ of the participants $(n=9)$ reported that the program's content had helped them "a lot" in dealing more effectively with their problems, and the remaining two participants reported that it had helped them "quite a bit".

Finally, the responses to the survey about their opinion of the treatment received were analyzed qualitatively. The selected analysis categories were: the objectives prior to the start of the intervention; the objectives achieved after the intervention; the impact of the group format on the participants; and overall satisfaction with the intervention. Table 3 shows the fundamental testimonies of some of the participants, which illustrate how they refer to these points, and the different categories that are framed within each one of them. 


\section{Discussion}

This study applies a psychological intervention based on improved emotion regulation, called UP, in a group format to a sample of females surviving domestic violence diagnosed with ED or subclinical symptoms that were users of the SCAIT service, a community service aimed at improving family well-being. We aimed to improve their clinical symptoms after the intervention (objectives 1 and 2), to maintain changes at 12-month follow-up (objective 3), and to obtain data about the feasibility, acceptance, and satisfaction with the UP (objective 4).

Preliminary results underscore the feasibility and clinical usefulness of the UP in group format applied in the social services context.

\section{Preliminary clinical utility data}

In this section, the results directly related to objectives 1 , 2 , and 3 will be addressed. When we assessed the participants' improvement in specific variables after treatment, the statistically significant increase in quality of life and the reduction of interference of symptomatology are promising. This fact is especially relevant in this sample, as the participants are subjected to intense psychosocial stressors, which sometimes do not cease with the passage of time. Despite not intervening directly on these stressors, the UP seems to improve the scores in these variables, being consistent with the results obtained in other studies (e.g., Osma et al. 2015). Specifically, the participants showed an improvement on the quality of life area of interpersonal relationships which includes family relationships, and within the subscales of the interference scale, they also had improved in the area of family life; which means that participants achieved one of their main objectives, which was to improve coexistence and family well-being after the UP intervention.

In addition, participants improved the emotion regulation skills, which is one of the specific aspects targeted by the UP and which is reflected in the statistically significant decrease in scores in total Difficulties in Emotion Regulation Scale punctuation. Specifically, a statistically significant reduction is observed for the subscales of rejection, interference and emotional lack of control $(p<0.001)$, but not for the lack of emotion attention and emotional confusion subscales $(p>0.05)$. The lack of significant results in these two specific subscales could be due to the fact that in the validation into Spanish the authors obtained internal consistency scores lower than the rest of the subscales, an aspect that the authors explain that could be due to the reduction of items for the adaptation and validation into Spanish (Hervás \& Jódar, 2008). Despite this, the significant reduction in emotional dysregulation, suggests the effectiveness of the UP to improve this variable (Mazaheri et al., 2014), an aspect that can have very positive consequences on parenting styles, promoting greater sensitivity in mothers and improving family well-being (Carreras et al., 2019). As for affective symptomatology, the results of this study show that the participants improved significantly in symptoms of panic, somatization, anxiety, and depression, thereby confirming the effectiveness of the UP to reduce emotional symptomatology (Sakiris \& Berle, 2019). Improving mothers' mental health can consequently decrease the probability that their children develop behavioral difficulties (D'Souza et al., 2019). Additionally, significant changes are also achieved in personality dimensions such as neuroticism and negative affect. These results are in line with those found in previous studies (e.g., Sauer-Zavala et al., 2012). In addition to improvements in negative affect and neuroticism, the application of the UP has achieved changes in extraversion, that is, the tendency to experience positive emotions such as satisfaction or happiness. Previous studies that have used the UP for the treatment of people with EDs have found contradictory results in this regard, as some of them confirm the changes in extraversion (e.g., Osma et al., 2015), and others do not (e.g., Ellard et al., 2010). More studies should be conducted to clarify these results on extraversion and positive affect.

In general, almost all the participants improved their scores after the intervention in the expected direction, and we highlight the overall improvement in some variables such as interference of symptomatology in their daily life, self-esteem, or quality of life. However, there are specific cases where improvement was not so obvious, and which are worth commenting on in more detail. An example is the case of Participant 2, who showed no significant changes. In this case, the fact that she suffers from fibromyalgia, a disease that causes high levels of pain and interferes considerably in her working life, may have influenced the results. Content customization (e.g., what variables influence the intensity of pain) and specific skills (e.g., mindfulness exercises focused on pain observation) in these cases could help improve the results.

Another case is that of Participant 6, who improved in almost all the variables after treatment but showed a significant worsening in positive affect at the 1-year followup. A possible explanation is that at the time of the1-year assessment, she had been evicted from her home, and had to find a new home for herself and her daughter, with few financial resources, which implied a very high level of stress. The last relevant case is that of Participant 11, who, although she showed significant changes in most variables 
after treatment, barely maintained these changes at the 1-year follow-up. This fact may have been due to problems caused by living with her daughter, who was diagnosed with borderline personality disorder and returned to live at home during that period of time. This daughter had spent some time in a child protection center for child-parental assault.

However, it is important to note that the cases of these three participants tend to be the most common among survivors of violence. As mentioned in the introduction, the number of moderating variables that can contribute to the impact of violence is immense (Galovski et al., 2021), so that an intervention can often result in limited progress due to all these moderating variables. In these cases, medical problems and family dynamics have an important influence and could be framed as factors that impact exposure to the trauma of violence (Helfrich et al., 2008; Herschell et al., 2017).

\section{Preliminary feasibility data}

In this section, the results directly related to objective 4 will be addressed. Regarding feasibility, the results of this study show that the users accepted this type of intervention, as the attendance rate was $82.64 \%$, well above those reported in other studies aimed at treating EDs in this population. For example, the CBT-based intervention for female survivors of violence by Johnson et al. (2011) reported an average attendance at 6.8 sessions out of $12(S D=4.3)$, representing an attendance rate of $56.66 \%$. In addition, no participant dropped out of our treatment. This information contrasts with studies that claim a general problem with adherence to psychological intervention programs in female victims of violence, shown in the high dropout rates, up to $66.98 \%$ (Hansen et al., 2014). We believe that the differential aspect of this group that made adherence so high and led to no dropouts was the fact that they had previously been users of the SCAIT and knew both the service and the staff. They came to this resource voluntarily and had already had positive experiences with other services. In addition, the group format enhanced feeling understood and interpersonal validation. Furthermore, the fact that the intervention was conducted in a community context rather than in a mental health unit might imply less stigma.

Another aspect that reflects the feasibility of the UP are the participants' responses to the satisfaction questionnaire, stating that the quality of the treatment program received is excellent and that it met their expectations, that they would recommend it to a loved one who was in the same situation and would even choose it again if they had to seek help once more. These results are similar to those found with the same instrument by Osma et al. (2019) in their UP application study for the transdiagnostic treatment of EDs in public mental health units in Spain. In addition, in the component rating questionnaires, the scores are very high for the protocol in general, with a mean score of 9 (score range: 9 out of 10). Psychoeducation about emotions and mindfulness techniques were the most valued components regarding its usefulness. The open answers provided by the participants (Table 3) also indicated that they have learned useful emotional strategies, they have achieved meaningful aims and they are satisfied with the intervention.

\section{Strengths and limitations}

One of the strengths of this study is to apply an emotionregulation based psychological intervention to treat EDs or subclinical affective symptomatology in female survivors of violence who are cared for in a community context to improve family well-being. This idea arises from the current need to resort to contexts in which psychological interventions can reach more people, rather than waiting for the people who are suffering to go to specialized clinical contexts (e.g., Bentley et al., 2017). The dissemination of evidence-based interventions in all sectors and contexts of society is essential if we are to reduce the emotional and economic costs arising from the main public health problems (Osma \& Sauer-Zavala, 2019), in this case, violence against women and the consequences of violence for all family members.

However, this study has some limitations. The first refers to the small sample size $(N=11)$. In this sense, we note that case and pilot studies are an advisable cost-effective method to study preliminarily the effectiveness, feasibility, and/or implementation of recent intervention programs in contexts other than those studied previously, but their results should be assessed prudently, and as a preliminary phase. Therefore, it would be interesting to replicate the results with a larger sample of women and through a randomized clinical trial. Another limitation is the lack of the incorporation of partners and/or family into the sessions, either at a specific moment or in a specific parallel intervention. A third limitation is that some of the measures used in the study did not have good internal consistency, which makes it more difficult to fully understand how the intervention has affected the constructs with less consistency. It would be necessary to replicate the analyses with a larger sample, to see if the internal consistency would improve. Given the nature of the violence experienced by women and the community context in which it has taken place, including family interventions could have contributed to an understanding of the participants' emotional problems and the possible reduction of interpersonal problems that can hinder their recovery. Finally, we could not develop some working material (participant's workbook) adapted to the nature and characteristics of the specific problem of violence against women, which would have led to greater customization of the intervention. Despite these limitations, the participants' rate of program attendance was not affected. 
As can be seen with this study, we have been able to preliminary explore two of the three axes proposed in the three-axis analysis (Ferro \& Vives, 2004). Thus, through objectives 1,2 , and 3 , we explore the clinical utility of the intervention and, therefore, the axis of efficacy, and, through objective 4 , the acceptability and viability of the intervention, that is, the axis of effectiveness. However, one of the main limitations is that due to the small sample size and the fact that it is a pilot study, no cost-effectiveness analyses have been carried out to explore the effectiveness axis. Therefore, and taking this limitation into account, it will be interesting for future studies to take this approach into account, in order to replicate the results that explore the axes, adding analyses that allow the exploration of effectiveness.

This study is added to the growing literature that supports the effectiveness and versatility of the UP applied in different contexts and for different populations. Having preliminary data on the feasibility and clinical usefulness of the UP for the treatment of EDs in females who have experienced violence, and in a context such as social services, can have countless advantages. The first is to be able to serve this group from a community approach, that is, with an interdisciplinary team that can evaluate and intervene comprehensively in this social problem. The second is the reduction of costs both for female users (e.g., traveling to a single resource) as well as for professionals (e.g., facilitating data collection and management). The third is the group application format, as it favors positive social reinforcement, modeling, and enhanced motivation for change and learning of better coping skills (Echeburúa et al., 2014). Thus, we consider that this type of intervention allows addressing the needs of this population more directly, offering them comprehensive care and improving their mental health from a biopsychosocial model, also having indirect positive consequences on other members of the family or partners of women, and thus improving family well-being.

\section{Conclusions}

With this study, we have obtained preliminary data showing the clinical utility and the viability and acceptability of UP in group format and with women who have experienced domestic violence. More specifically, the MANOVA results showed an increase in quality of life, self-esteem, and extraversion, as well as a reduction in interference, neuroticism, somatization, anxiety, emotional discontent, negative affect, depression, and emotional rejection. Regarding acceptability, a high attendance rate and no dropouts were obtained. However, it must be taken into account that this is a pilot study, and, therefore, it has several limitations that mean that we must be cautious in interpreting the results. Among these limitations, we should include small sample sizes, the fact that some measures did not obtain high internal consistency, the fact that the efficiency axis could not be analyzed by means of cost-effectiveness analysis, the inability to introduce partners or relatives into the group, the unfeasibility to carry out family intervention sessions, or the failure to develop working material such as a manual for the participants. Taking into account that the results are promising, the need for randomized controlled clinical studies is justified to overcome the limitations of the present study. In this sense, future lines should fundamentally value introducing partners or families to the intervention, providing material for the participants, and carrying out more controlled analyses with a larger sample. All this to replicate the analyses and establish a greater exploration of the three axes of efficacy, efficiency, and effectiveness. Having data such as the one in this study could make it possible to address the needs of this population in a more direct way, offering them comprehensive care, and improving their mental health from a biopsychosocial model. Likewise, this makes it possible to indirectly improve the well-being of the rest of the family members.

Acknowledgements We are grateful for the collaboration and effort of all the professionals of the SCAIT of Social Services Center of Benicarló, the Hospital Comarcal de Vinaròs, and especially all the participants who made this study possible. We also thank the Ministry of Equality and Inclusive Policies of the Generalitat Valenciana for awarding this study first prize in the "Amparo Moreno Vañó Awards" for innovation in the field of social services, that underlines the need to implement new methods of intervention that have been effective in other contexts into social services.

Funding Funding for the study was provided by Gobierno de Aragón (Departamento de Innovación, Investigación y Universidad) and FEDER "Construyendo Europa desde Aragón" (research team S31_20D). Open Access funding provided thanks to the CRUE-CSIC agreement with Springer Nature.

\section{Compliance with Ethical Standards}

Conflict of Interest The authors declare no competing interests.

Publisher's note Springer Nature remains neutral with regard to jurisdictional claims in published maps and institutional affiliations.

Open Access This article is licensed under a Creative Commons Attribution 4.0 International License, which permits use, sharing, adaptation, distribution and reproduction in any medium or format, as long as you give appropriate credit to the original author(s) and the source, provide a link to the Creative Commons license, and indicate if changes were made. The images or other third party material in this article are included in the article's Creative Commons license, unless indicated otherwise in a credit line to the material. If material is not included in the article's Creative Commons license and your intended use is not permitted by statutory regulation or exceeds the permitted use, you will need to obtain permission directly from the copyright holder. To view a copy of this license, visit http://creativecommons. org/licenses/by/4.0/. 


\section{Appendix 1}

\section{Questionnaire for the evaluation of the Unified Protocol components (Osma, Crespo, Fermoselle, Sala and Castellano, 2014)}

Name (code):

Date:

\begin{tabular}{|l|l|}
\hline Post-treatment & \\
\hline 3-month follow-up & \\
\hline 6-month follow-up & \\
\hline 12-month follow-up & \\
\hline
\end{tabular}

Point of Assessment (Mark with a cross):

1. To what extent do you consider that the therapy you received has helped you adaptively regulate your emotions?

\begin{tabular}{|c|c|c|c|c|c|c|c|c|c|c|}
\hline 0 & 1 & 2 & 3 & 4 & 5 & 6 & 7 & 8 & 9 & 10 \\
\hline Nothing & & & & & & & & & & A lot \\
\hline
\end{tabular}

2. Of the techniques and exercises that we practice during therapy, to what extent do you consider that each of them has helped you to regulate your emotions properly?

- Identify the three-component of emotional experience: Thoughts, Physical Sensations and Behavior.

\begin{tabular}{|c|c|c|c|c|c|c|c|c|c|c|}
\hline 0 & 1 & 2 & 3 & 4 & 5 & 6 & 7 & 8 & 9 & 10 \\
\hline None & & & & & & & & & & A lot \\
\hline
\end{tabular}

- Analyze the ARC of emotions: Antecedents, Responses, and Consequences.

\begin{tabular}{|c|c|c|c|c|c|c|c|c|c|c|}
\hline 0 & 1 & 2 & 3 & 4 & 5 & 6 & 7 & 8 & 9 & 10 \\
\hline None & & & & & & & & & & A lot \\
\hline
\end{tabular}

- $\quad$ Emotional awareness, present-focused, nonjudgmental.

\begin{tabular}{|c|c|c|c|c|c|c|c|c|c|c|}
\hline 0 & 1 & 2 & 3 & 4 & 5 & 6 & 7 & 8 & 9 & 10 \\
\hline None & & & & & & & & & & A lot \\
\hline
\end{tabular}

- Identify automatic appraisals ("jumping to conclusions" and "thinking the worst").

\begin{tabular}{|c|c|c|c|c|c|c|c|c|c|c|}
\hline 0 & 1 & 2 & 3 & 4 & 5 & 6 & 7 & 8 & 9 & 10 \\
\hline None & & & & & & & & & & A lot \\
\hline
\end{tabular}

- Carry out the cognitive re-evaluation and flexibility.

\begin{tabular}{|c|c|c|c|c|c|c|c|c|c|c|}
\hline 0 & 1 & 2 & 3 & 4 & 5 & 6 & 7 & 8 & 9 & 10 \\
\hline None & & & & & & & & & & A lot \\
\hline
\end{tabular}

- Identify emotional avoidance strategies.

\begin{tabular}{|c|c|c|c|c|c|c|c|c|c|c|}
\hline 0 & 1 & 2 & 3 & 4 & 5 & 6 & 7 & 8 & 9 & 10 \\
\hline None & & & & & & & & & & A lot \\
\hline
\end{tabular}

- Generate alternative actions to Emotion-Driven Behaviors (EDB).

\begin{tabular}{|c|c|c|c|c|c|c|c|c|c|c|}
\hline 0 & 1 & 2 & 3 & 4 & 5 & 6 & 7 & 8 & 9 & 10 \\
\hline None & & & & & & & & & & A lot \\
\hline
\end{tabular}

- Induce physical sensations.

\begin{tabular}{|c|c|c|c|c|c|c|c|c|c|c|}
\hline 0 & 1 & 2 & 3 & 4 & 5 & 6 & 7 & 8 & 9 & 10 \\
\hline None & & & & & & & & & & A lot \\
\hline
\end{tabular}

- Carry out emotional exposures.

\begin{tabular}{|c|c|c|c|c|c|c|c|c|c|c|}
\hline 0 & 1 & 2 & 3 & 4 & 5 & 6 & 7 & 8 & 9 & 10 \\
\hline None & & & & & & & & & & A lot \\
\hline
\end{tabular}




\section{References}

Andreu, L., Galdón, M. J., Dura, E., Fernando, M., Murgui, S., García, A., \& Ibáñez, E. (2008). Psychometric properties of the Brief Symptoms Inventory-18 (BSI-18) in a Spanish sample of outpatients with psychiatric disorders. Psicothema, 20(4), 844-850.

Barlow, D. H., Farchione, T. J., Bullis, J. R., Gallagher, M. W., Murray-Latin, H., Sauer-Zavala, S., Bentley, K. H., ThompsonHollands, J., Conklin, L. R., Boswell, J. F., Ametaj, A., Carl, J. R., Boettcher, H. T., \& Cassiello-Robbins, C. (2017). The unified protocol for transdiagnostic treatment of Emotional Disorders compared with diagnosis-specific protocols for anxiety disorders: A randomized clinical trial. JAMA Psychiatry, 74(9), 875-884. https://doi.org/10.1001/jamapsychiatry.2017.2164.

Barlow, D. H., Farchione, T. J., Sauer-Zavala, S., Murray-Latin, H., Ellard, K. K., Bullis, J. R., Bentley, K. H., Boettcher, H. T., \& Cassiello-Robbins, C. (2018). Unified protocol for transdiagnostic treatment of emotional disorders: Therapist guide. 2nd ed. New York, NY: Oxford University Press.

Barlow, D. H., Sauer-Zavala, S., Carl, J. R., Bullis, J. R., \& Ellard, K. K. (2014). The nature, diagnosis, and treatment of neuroticism back to the future. Clinical Psychological Science, 2(3), 344-365. https://doi.org/10.1177/2167702613505532.

Bentley, K. H., Boettcher, H., Bullis, J. R., Carl, J. R., Conklin, L. R., Sauer-Zavala, S., Pierre-Louis, C., Farchione, T. J., \& Barlow, D. H. (2017). Development of a single-session, transdiagnostic preventive intervention for young adults at risk for emotional disorders. Behavior Modification, 42(5), 781-805. https://doi.org/ 10.1177/0145445517734354.

Boeckel, M. G., Blasco-Ros, C., Grassi-Oliveira, R., \& Martínez, M. (2014). Child abuse in the context of intimate partner violence against women: the impact of women's depressive and posttraumatic stress symptoms on maternal behavior. Journal of Interpersonal Violence, 29(7), 1201-1227. https://doi.org/10. $1177 / 0886260513506275$

Boettcher, H. T., \& Conklin, L. R. (2017). Transdiagnostic assessment and case formulation: Rationale and application with de unified protocol. In D. H. Barlow \& T. J. Farchione (Eds.), Applications of the unified protocol for transdiagnostic treatment of emotional disorders (pp. 17-37). New York, NY: Oxford University Press.

Bradley, F., Smith, M., Long, J., \& O’Dowd, T. (2002). Reported frequency of domestic violence: Cross sectional survey of women attending general practice. British Medical Journal, 324(7332), 271-276.

Briere, J. \& \& Jordan, C. E. (2004). Violence against women: Outcome complexity and implications for assessment and treatment. Journal of Interpersonal Violence, 19(11), 1252-127. https://doi. org/10.1177/0886260504269682.

Brown, N., Wojtalik, J. A., Turkel, M., Vuper, T., Strasshofer, D., Sheline, Y. I., \& Bruce, S. E. (2016). Neuroticism and Its associated brain activation in women with PTSD. Journal of Interpersonal Violence, 35(1-2), 341-363. https://doi.org/10.1177/ 0886260516682519.

Brown, T. A., \& Barlow, D. H. (2009). A proposal for a dimensional classification system based on the shared features of the DSM-IV anxiety and mood disorders: Implications for assessment and treatment. Psychological assessment, 21(3), 256-271.

Brown, T. A., Campbell, L., Lehman, C., Grisham, J., \& Mancill, R. (2001). Current and lifetime comorbidity of the DSM-IV anxiety and mood disorders in a large clinical sample. Journal of Abnormal Psychology, 110(4), 585-599. https://doi.org/10.1037/ 0021-843X.110.4.585.

Buesa, S., \& Calvete, E. (2013). Violencia contra la mujer y síntomas de depresión y estrés postraumático: el papel del apoyo social.
International Journal of Psychology and Psychological Therapy, 13(1), 31-45.

Bullis, J. R., Boettcher, H., Sauer-Zavaa, S., \& Barlow, D. H. (2019). What is an emotional disorder? A transdiagnostic mechanistic definition with implications for assessment, treatment and prevention. Clinical Psychology Science and Practice, 26(2), 1-19. https://doi.org/10.1111/cpsp.12278.

Carreras, J., Carter, A. S., Heberle, A., Forbes, D., \& Gray, S. A. O. (2019). Emotion regulation and parent distress: getting at the heart of sensitive parenting among parents of preschool children experiencing high sociodemographic risk. Journal of Child and Family Studies, 28(11), 2953-2962. https://doi.org/10.1007/ s10826-019-01471-z.

Christopher, C., Saunders, R., Jacobvitz, D., Burton, R., \& Hazen, N. (2013). Maternal empathy and changes in mothers' permissiveness as predictors of toddlers' early social competence with peers: A parenting intervention study. Journal of Child and Family Studies, 22(6), 769-778. https://doi.org/10.1007/ s10826-012-9631-z.

Consellería de Bienestar Social. (2014). Protocolo de actuación de los SEAFI: Servicios especializados de atención a menores en riesgo o con medidas jurídicas de protección y a sus familias. Valencia: Generalitat Valenciana.

Costa, P. T., \& McCrae, R. R. (1999). Revised NEO Personality Inventory (NEO-PI-R) and NEO Five-Factor Inventory (NEO$F F I)$. Madrid: TEA Ediciones.

D'Souza, S., Underwood, L., Peterson, E. R., Buckley, J., Morton, S. M. B., \& Waldie, K. E. (2019). Determinants of persistence and change in early childhood behavioural problems: The roles of parenting and maternal mental health. Journal of Child and Family Studies, 28(7), 1826-1842. https://doi.org/10.1007/ s10826-019-01404-w.

De Andrés Pizarro, J. (2000). El análisis de estudios cualitativo. Atención Primaria, 25(1), 42-46. https://doi.org/10.1016/s02126567(00)78463-0.

Derogatis, L. R. (2001). Brief Symptom Inventory (BSI)-18. Administration, scoring and procedures manual. Minneapolis: NCS Pearson, Inc.

Echeburúa, E., De Corral, P., \& Fernández-Montalvo, J. (2000). Escala de inadaptación (EI): Propiedades psicométricas en contextos clínicos. Análisis y Modificación de Conducta, 26(107), 325-340.

Echeburúa, E., Sarasua, B., \& Zubizarreta, I. (2014). Individual versus individual and group therapy regarding a cognitive-behavioral treatment for battered women in a community setting. Journal of Interpersonal Violence, 29(10), 1783-1801. https://doi.org/10. $1177 / 0886260513511703$.

Ellard, K. K., Fairholme, C. P., Boisseau, C. L., Farchione, T. J., \& Barlow, D. H. (2010). Unified protocol for the transdiagnostic treatment of emotional disorders: Protocol development and initial outcome data. Cognitive and Behavioral Practice, 17, 88-101. https://doi.org/10.1016/j.cbpra.2009.06.002.

Ferrando, L., Bobes, J., Gibert, J., Soto, M., \& Soto, O. (2000). MINI entrevista neuropsiquiátrica internacional. Madrid: Instituto IAP.

Ferro, R., \& Vives, M. (2004). Un análisis de los conceptos de efectividad, eficacia y eficiencia en psicología.Panace@: Revista de Medicina, Lenguaje y Traducción, 5(16), 97-99.

Galovski, T. E., Werner, K. B., Iverson, K. M., Kaplan, S., Fortier, C. B., Fonda, J. R., \& McGlinchey, R. E. (2021). A multi-method approach to a comprehensive examination of the psychiatric and neurological consequences of intimate partner violence in women: A methodology protocol. Frontiers in Psychiatry, 12, 108 https://doi.org/10.3389/fpsyr.2021.569335.

Gobierno de España [Spanish Govt.]. (2019). Portal Estadístico de la Delegación del Gobierno para la Violencia de Género. Retrieved from http://estadisticasviolenciagenero.igualdad.mpr.gob.es/. 
Graham-Bermann, S., Sularz, A. R., \& Howell, K. H. (2011). Additional adverse events among women exposed to intimate partner violence: Frequency and impact. Psychology of Violence, 1(2), 136-149. https://doi.org/10.1037/a0022975.

Gratz, K. L., \& Roemer, L. (2004). Multidimensional assessment of emotion regulation and dysregulation: Development, factor structure, and initial validation of the difficulties in emotion regulation scale. Journal of Psychopathology and Behavioral Assessment, 26(1), 41-54.

Hansen, N. B., Eriksen, S. B., \& Elklit, A. (2014). Effects of an intervention program for female victims of intimate partner violence on psychological symptoms and perceived social support. European Journal of Psychotraumatology, 5, 1-10. https://doi. org/10.3402/ejpt.v5.24797.

Helfrich, C. A., Fujiura, G. T., \& Rutkowski-Kmitta, V. (2008). Mental health disorders and functioning of women in domestic violence shelters. Journal of Interpersonal Violence, 23(4), 437-453. https://doi.org/10.1177/0886260507312942.

Herschell, A. D., Scudder, A. B., Schaffner, K. F., \& Slagel, L. A. (2017). Feasibility and effectiveness of parent-child interaction therapy with victims of domestic violence: A pilot study. Journal of Child and Family Studies, 26(1), 271-283. https://doi.org/10. 1007/s10826-016-0546-y.

Hervás, G., \& Jódar, R. (2008). The Spanish version of the difficulties in emotion regulation scale. Clinica Y Salud, 19(2), 139-156.

IBM Corp. (2013). IBM SPSS Statistics for Windows, Version 22.0. New York, NY: IBM Corp.

Institute for Health Metrics and Evaluation. (2017). Global Health Data Exchange. Retrieved from http://ghdx.healthdata.org/gbdresults-tool.

Jacobson, N. S., \& Truax, P. (1991). Clinical significance: A statistical approach to defining meaningful change in psychotherapy research. Journal of Consulting and Clinical Psychology, 59, 12-19.

Johnson, D. M., Zlotnick, C., \& Perez, S. (2011). Cognitive-behavioral treatment of PTSD in residents of battered women shelters: Results of a randomized clinical trial. Journal of Consulting and Clinical Psychology, 79(4), 542-551. https://doi.org/10.1038/jid. 2014.371.

Kamody, R. C., Howell, K. H., Schwartz, L. E., Schaefer, L. M., \& Thurston, I. B. (2020). A cross-sectional examination of intimate partner violence and mother-child communication. Journal of Child and Family Studies, 29(5), 1363-1373. https://doi.org/10. 1007/s10826-019-01617-z.

Kennedy, K. A., \& Barlow, D. H. In: In D. H. Barlow T. J. Farchione, (eds.) 2017). The unified protocol for transdiagnostic treatment of emotional disorders: An introduction. Applications of the unified protocol for transdiagnostic treatment of emotional disorders. (pp. 1-16). New York, NY: Oxford University Press.

Larizgoitia, I. (2006). La violencia también es un problema de salud pública. Gaceta Sanitaria, 20(1), 63-70. https://doi.org/10.1157/ 13086028.

Mak, M. C. K., Yin, L., Li, M., Cheung, R. Y. H., \& Oon, P. T. (2020). The Relation between parenting stress and child behavior problems: Negative parenting styles as mediator. Journal of Child and Family Studies, 29, 2993-3003. https://doi.org/10.1007/ s10826-020-01785-3.

Mazaheri, M., Daghaghzadeh, H., Afshar, H., \& Mohammadi, N. (2014). The effectiveness of the unified protocol on emotional dysregulation and cognitive emotion regulation strategies in patients with psychosomatic disorders. International Journal of Body, Mind \& Culture, 1(1), 73-82.

Mezzich, J. E., Ruipérez, M. A., Pérez, C., Yoon, G., Liu, J., \& Mahmud, S. (2000). The Spanish version of the quality of life index. The Journal of Nervous and Mental Disease, 188(5), 301-305.
Miller-Graff, L. E., \& Howell, K. H. (2016). Interventions for preventing violence and remediating its negative effects: Contemporary priorities and future directions. Psychology of Violence, 6(3), 361-367. https://doi.org/10.1037/vio0000058.

Ministerio de Sanidad y Consumo (2003). Volencia Doméstica. Promoción de la Salud y Epidemiología. Retrieved from: https://www.mscbs.gob.es/ca/ciudadanos/violencia/docs/ VIOLENCIA_DOMESTICA.pdf

Muñoz, M. (2009). Estigma y enfermedad mental. Análisis del rechazo social que sufren las personas con enfermedad mental. Madrid: Editorial Complutense.

Osma, J., \& Sauer-Zavala, S. (2019). Protocolo Unificado: líneas futuras y nuevos retos. In J. Osma (Ed.), Aplicaciones del Protocolo Unificado para el tratamiento transdiagnóstico de la disregulación emocional (pp. 319-332). Madrid: Alianza Editorial.

Osma, J., Castellano, C., Crespo, E., \& García-Palacios, A. (2015). The Unified Protocol for transdiagnostic treatment of emotional disorders in group format in a Spanish public mental heath setting. Behavioral Psychology, 23(3), 447-466.

Osma, J., Peris-Baquero, O., Suso-Ribera, C., Farchione, T. J. \& Barlow, D. H. (2021). Effectiveness of the Unified Protocol for transdiagnostic treatment of emotional disorders in group format in Spain: Results from a randomized controlled trial with 6-months follow-up. Psychotherapy Research, 16, 1-14. https:// doi.org/10.1080/10503307.2021.1939190.

Osma, J., Suso-Ribera, C., \& Peris-Baquero, O. (2019). Eficacia del Protocolo Unificado en formato grupal para el tratamiento transdiagnóstico de los trastornos emocionales en unidades de salud mental públicas españolas. In J. Osma (Ed.), Aplicaciones del Protocolo Unificado para el tratamiento transdiagnóstico de la disregulación emocional (pp. 203-219). Madrid: Alianza Editorial.

Overstreet, S. (2000). Exposure to community violence: Defining the problem and understanding the consequences. Journal of Child and Family Studies, 9(1), 7-25. https://doi.org/10.1023/A: 1009403530517.

Paz, P., Labrador, F. J., Arinero, M., \& Crespo, M. (2004). Efectos psicopatológicos del maltrato doméstico. Avances en Psicología Latinoamericana, 22, 105-116.

Rosenberg, M. (1965). La autoimagen del adolescente y la sociedad. Buenos Aires: Paidos (Traducción al español de 1973).

Sakiris, N., \& Berle, D. (2019). A systematic review and meta-analysis of the Unified Protocol as a transdiagnostic emotion regulation based intervention. Clinical Psychology Review, 72, 1017-1051. https://doi.org/10.1016/j.cpr.2019.101751.

Sandín, B., Chorot, P., Lostao, L., Joiner, T. E., Santed, M. A., \& Valiente, R. M. (1999). Escalas PANAS de Afecto Positivo y Negativo: validación factorial y convergencia transcultural. Psicothema, 11(1), 37-51.

Sauer-Zavala, S., Boswell, J. F., Gallagher, M. W., Bentley, K. H., Ametaj, A., \& Barlow, D. H. (2012). The role of negative affectivity and negative reactivity to emotions in predicting outcomes in the unified protocol for the transdiagnostic treatment of emotional disorders. Behaviour Research and Therapy, 50, 551-557. https://doi.org/10.1016/j.brat.2012.05.005.

Sauer-Zavala, S., Cassiello-Robbins, C., Ametaj, A. A., Wilner, J. G., \& Pagan, D. (2019). Transdiagnostic treatment personalization: The feasibility of ordering unified protocol modules according to patient strengths and weaknesses. Behavior Modification, 43(4), 518-543. https://doi.org/10.1177/0145445518774914.

Sauer-Zavala, S., Cassiello-Robbins, C., Conklin, L. R., Bullis, J. R., Thompson-Hollands, J., \& Kennedy, K. A. (2017). Isolating the unique effects of the unified protocol treatment modules using single case experimental design. Behavior Modification, 41(2), 286-307. https://doi.org/10.1177/0145445516673827. 
Sheehan, D. V., Lecrubier, Y., Sheehan, K. H., Amorim, P., Janavs, J., Weiller, E., Hergueta, T., Baker, R., \& Dunbar, G. C. (1998). The Mini-International Neuropsychiatric Interview (M. I.N.I): The development and validation of a structured diagnostic psychiatric interview for DSM-IV and ICD-10. The Journal of Clinical Psychiatry, 59, 20.

Sospedra, R., Molero, R. J., Moral, M. J., Albiñana, P., \& Sabater, Y. (2010). Los SEAFI's (Servicios Especializados de Atención a la Familia e Infancia), un recurso de intervención dirigido a familias en situación de vulnerabilidad o conflicto. International Journal of Developmental and Educational Psychology.

Trabold, N., McMahon, J., Alsobrooks, S., Whitney, S., \& Mittal, M. (2018). A systematic review of intimate partner violence interventions: State of the field and implications for practitioners. Trauma, violence \& Abuse, 1-15. https://doi.org/ $10.1177 / 1524838018767934$.

Vázquez, A. J., García-Bóveda, R., \& Vázquez-Morejón, R. (2004). Escala de autoestima de Rosenberg: Fiabilidad y validez en población clínica española. Apuntes de Psicología, 22(2), 247-255.

Watson, D., Clark, L. A., \& Tellegen, A. (1988). Development and validation of brief measures of positive and negative affect: The PANAS scales. Journal of Personality and Social Psychology, 54, 1063-1070.

World Health Organization. (2017a). Depression and other common mental disorders: Global health estimates. Ginebra: World Health Organization.

World Health Organization. (2017b). Violencia contra la mujer [Violence against women]. Retrieved from https://www.who.int/ es/news-room/fact-sheets/detail/violence-against-women. 